\title{
La fracture ostéoporotique de l'extrémité supérieure du fémur : un modèle accompli de fragilité, reflet de la faillite des réserves fonctionnelles chez le sujet âgé
}

\author{
C. Jeandel \\ (C) Springer-Verlag 2009
}

L'ostéoporose, caractérisée par une diminution de la masse osseuse et des altérations de l'architecture osseuse responsables d'une fragilité osseuse, et ses conséquences fracturaires vont représenter, dans les années à venir, un véritable enjeu de santé publique. À l'âge de 80 ans, $70 \%$ des femmes présentent une ostéoporose selon les critères densitométriques ( $T$-score inférieur à -2,5), mesurée à la hanche ou à l'avant-bras, et $60 \%$ des femmes ostéoporotiques seront victimes d'au moins une fracture par fragilité osseuse. Le nombre des fractures de hanche et leurs coûts associés devraient ainsi s'accroître massivement et pourraient tripler d'ici à 2040. Les coûts directs des fractures ostéoporotiques ont ainsi été estimés à 13,8 milliards de dollars aux États-Unis, alors qu'ils s'élevaient de cinq à six milliards dix ans auparavant. Plus fréquente chez la femme après la ménopause, l'ostéoporose et ses complications vont également concerner de plus en plus l'homme âgé. Si le risque de fracture de hanche tout au long de la vie est ainsi estimé à 17,5\% chez les femmes, il est voisin de $6 \% \mathrm{chez}$ les hommes. La prévalence chez le sujet âgé de certaines comorbidités et la fréquence de prescription de thérapeutiques médicamenteuses potentiellement ostéopéniantes ou interagissant avec le métabolisme osseux (corticoïdes, antiépileptiques, IPP, analogues de la GnRH, anti-aromatases, etc.) rend compte de la place croissante des causes d'ostéoporose secondaires chez le sujet âgé et de la prévalence croissante de l'affection chez l'homme. Parmi ces morbidités, certaines exercent un effet additionnel sur le risque fracturaire tel que la dénutrition protéinoénergétique qui avec le déconditionnement physique expose au risque de sarcopénie et de chute, et tel que l'insuffisance rénale qui majore le risque de déficience en vitamine $\mathrm{D}$ et donc de composante malacique associée. La fracture de l'extrémité supérieure du fémur témoigne le plus souvent de la faillite des réserves fonctionnelles : résistance osseuse, puissance et force musculaire, maintien du contrôle postural, réserves cognitives, etc. Elle est pour cette raison assortie d'un mauvais pronostic fonctionnel et représente une des causes prévalentes de dépendance aggravée mais grève également le pronostic vital de par ses complications (syndrome d'immobilisation, plaies de pression, maladie thromboembolique, infections, etc.). Les progrès accomplis dans la compréhension des mécanismes physiopathologiques font désormais de l'ostéoporose fracturaire une maladie à part entière et ont permis l'émergence de nouvelles familles et pistes thérapeutiques devant permettre dans le cadre d'une prise en charge globale d'en réduire l'incidence.

Le dossier thématique de ce numéro des Cahiers de l'année gérontologique est donc consacré à ce domaine de la médecine pour lequel le médecin généraliste et le gériatre vont être de plus en plus concernés. Il sera complété dans le prochain numéro par des articles de mise au point abordant les stratégies diagnostiques et thérapeutiques dans l'ostéoporose du sujet âgé.

C. Jeandel $(\bowtie)$

Centre de prévention et de traitement des maladies

du vieillissement, pôle de gérontologie,

CHU de Montpellier, avenue Charles-Flahault,

F-34295 Montpellier cedex, France

e-mail : c-jeandel@chu-montpellier.fr 